\title{
Performance Evaluation of Handover Channel Exchange Scheme in GSM Network
}

\author{
Onyishi D.U \\ Department of Electrical \& \\ Electronics Engineering, \\ Federal University of \\ Petroleum Resources Effurun
}

\author{
Nwalozie G.C and Aniedu \\ A.N \\ Department of Electronic \& \\ Computer Engineering \\ Nnamdi Azikiwe University \\ Awka, Anambra State
}

\author{
Ejiofor A.C \\ Department of Industrial \\ production Engineering \\ Nnamdi Azikiwe University \\ Awka, Anambra State
}

\begin{abstract}
Properly designed handover procedure in cellular wireless systems are essential for maintaining continuity of a call in progress and minimizing the probability of forced termination, signaling and switching load on the network. In this research, the Handover Channel Exchange (HCE) scheme is modeled and analyzed for channels between two mobiles that are moving in opposite directions across the handover area of adjacent cells of a GSM network. The channel exchange method is interesting since it yields low values of handover failure probability as compared to the case with no channel exchange. This work compares the performance of the cellular system with and without channel exchange, using the data obtained from a GSM network, with MATLAB as the platform for the simulation and analysis. The results as presented show improvement in handover failure probability obtained by using the channel exchange as against that of no channel exchange obtained from the GSM network
\end{abstract}

General Terms: failure Probability, Handover Channel Exchange, call arrival rate.

\section{INTRODUCTION}

Mobility is one of the most important features of a wireless communication system. Usually, continues service is achieved by supporting handover from one cell to another. In present day cellular systems where the trend is towards smaller cell sizes or microcell so as to increase capacity, the handover procedure adopted in the system has a significant impact on the overall performance of the system. Poorly designed handover scheme tend to generate very heavy signaling traffic and thereby, a dramatic decrease in the quality of service (QOS).

A good handover procedure is essential in maintaining continuity of a call in progress and keeping as low as possible the probability of forced termination of calls, the new call blocking probability and signaling and switching load on the network. The probability of forced termination of call is the probability of dropping an active call due to a handover failure. New call blocking probability is the probability of blocking a new call due to non-availability of a free channel. Since it was stated that the quality of service (QOS) of a communication system is affected by the handover technique it employs. Then it is important and worthwhile to propose a scheme that reduces the unwanted dropping of calls and also reduces the signaling load on the network and invariably improve the quality of service of the network.

In technical literature, several handover policies and channel assignment policies have been proposed to minimize handover failures in mobile cellular communication. Salih and Fidanboyu [1], [2] described and modeled queuing techniques for two- tier cellular networks. In [1] a micro cell/macrocell network using a FIFO queue in macro cell tier and in [2] a microcell/macrocell network using a FIFO is queue in microcell tier is introduced and compared with each other. The result for both systems showed that forced termination probability for slow users is decreased when the FIFO queue is used in microcell and forced termination for fast users is decreased when queue is in macrocell.

Zhang and Liu [3] proposed an adaptive algorithm that assigns the number of channel adaptively. When forced termination probability exceeds a predefined threshold the guard channels number is increased to reduce the force termination probability below the threshold. The use of guard channels requires careful determination of the optimum number of these channels, knowledge of the traffic pattern of the area and estimations of channel occupancy time distribution.

In [4] a queuing scheme using guard channels is described in this, both new calls and handover calls are queued. A number of guard channel are reserved for handover calls, when the new calls are congested, a channel from the guard channels is used if it is available. This scheme decreases the call blocking probability while increasing forced termination probability slightly. In [5] a fast handover algorithm for hard handover is proposed to remove fast fading fluctuations resulting in algorithm that reacts more quickly to corner effect. The authors propose a technique called local averaging in which the averaging time interval is smaller than averaging time interval of common handover algorithm and improve handover performance.

A. Jera, A.Molinaro and S. Marano in [6] a bonus - based algorithm is proposed where it is compared with classical and macro algorithms. In the classical algorithm, in the case of new call request, a user is assigned to microcell or overflowed to macrocell if capacity of microcell is full. After the user speed estimation is done, the user is assigned to the appropriate layer using overflow and takes back. This scheme results in too many handovers known as ping - pong. Macro algorithm is similar to classical algorithm with one exception. When a user is assigned to the microcell it is not permitted to take-back to micro cell which decreases the number of handover. The bonus-based algorithm tries to prevent unnecessary handovers when fast users temporarily slow down .For each fast user a time bonus is given and users can use this time bonus during temporary slowdown. If a user exceeds the timer then it is assigned as a slow user and is taken -back to the microcell layer. 
The aim of research work is to present a detailed analysis of a hand over scheme (Handover Channel Exchange) which does not only reduce the probability of forced termination and signaling load on a network but at the same time without a significant increase in new call blocking probability.

\section{TYPES OF HANDOVER}

\subsection{Hard Handover and Soft Handover}

A hard handover is essentially a "break before make" connection. Under the control of the MSC, the BS hands over the MS's call to another cell and then drop the call. In a hard handover, the link to the prior BS is terminated before or as the user is transferred to the new cell's BS, the MS is linked to no more than one BS at any given time [7]. Hard handover is primarily used by systems, which use FDMA (frequency division multiple access) and TDMA (time division multiple access), where different frequency ranges are used in adjacent channels in order to minimize channel interference. So when the MS moves from one BS to another BS, it becomes impossible for it to communicate with both BSs (since different frequencies are used). In contrast to hard handover, a soft handover can establish multiple connections with neighboring cells. Soft handover is used by the code division multiple access (CDMA) systems where the cells use same frequency band using different code words.

\subsection{Multilayer Handover}

In practical cellular systems, several problems arise when designing handover scheme for a wide range of mobile velocities. High-speed users pass through the coverage area of cell within matter of a second, whereas pedestrian users may never need a handover during a call. The mobile switching center can quickly become over- burdened if high-speed users are constantly being passed between very small cells.

By using different antenna heights (often on the same tower) and different power levels, it is possible to provide large and small cells, which are co-located at a single location. The high-speed users are assigned to the large cell and low speed to the micro cell.

A number of microcells are overlaid by a macrocell and the users are assigned to each layer according to their speeds. Microcells and macrocells coverage area are respectively about 500 meters and $35 \mathrm{Km}$ for GSM900. Since slow users are assigned to the microcells and fast users are assigned to the macrocells, the total number of handover requests is decreased. Macro cells not only serve the fast users but also serve slow users when the microcells are congested. When a micro cell allocates all of its channels, the new and handover calls are overflowed to the macrocell layer. When the microcells load decreases it is possible to assign slow users back to the microcell. This type of handoff is called takeback.

\section{HANDOVER DECISION}

There are numerous methods for performing handover, at least as many as the kinds of state information that have been defined for MSs, as well as the kinds of network entities that maintain the state information. The decision- making process of handoff may be centralized or decentralized (i.e., the handover decision may be made at the MS or network). From the decision process point of view, one can find a least three different kinds of handoff decisions [8].

\subsection{Network - Controlled Handover}

In a network-controlled handover protocol, the makes a handoff decision based on the measurement of the MSs at a number of BSs. In general, the handover process (including data transmission, channel switching, and network switching) takes $100-200 \mathrm{~ms}$. Information about the signal quality for all users is available $t$ a single point in the network that facilitates appropriate resource allocation. Network - controlled handoff is used in first- generation analog systems such as AMPS (advanced mobile phone system) TACS (total access communication system)

\subsection{Mobile - Assisted Handover}

In a mobile-assisted process, the MS makes measurements and the network makes the decisions. In GSM, the BS subsystem includes a base transceiver station (BTS) and a base station controller (BSC). The BSC is in contact with MSs through the radio interface and includes radio transmission and receiver devices and signal processing. The BSC is in contact with the network and is in charge of the radio interface management, mainly the allocation and release of radio channels and handoff management. The handover time in GSM is approximately I second.

\subsection{Mobile - Controlled Handover}

In mobile-controller handover, each MS is completely in control of the handover process. This type of handover has a short reaction time (on the order of 0.1 second) .The MS measures the signal strengths from the surrounding BSS and interference levels on all channels. A handover can be initiated if the signal strength of the serving BS is lower than that of another BS by a certain threshold. Digital European Telephone (DECT) is an example of cellular system using MCHO.

\section{METHODOLOGY}

In this research a mathematical formulation of the proposed Handover Channel Exchange (HCE) scheme using a two-cell model is presented. The two-cell model is simulated with MATLAB. The result is validated by comparing it with data obtained from a GSM network (STARCOMMS NIG LTD), which does not implement the scheme. This provides a fast, easy and cost effective evaluation. The analytical approach gives insight into handover behaviour quickly while simulations are required for complex scenario. Hence, the combination of analytical and simulation approaches can be very powerful. The simulation model incorporates the following component: the cell model, propagation model, traffic model and mobility model.

* Cell model: the cell model used in the simulation and analysis is that of two- cell.

* Propagation model: The performance of wireless communication system depends significantly on the mobile radio channel. The radio waves propagate through the mobile radio channel through different mechanism such as reflection, diffraction and scattering.

* Traffic model: Traffic model in the project is assumed to be homogeneous and symmetrical, that is traffic is evenly distributed among the cells.

* Mobility model: The mobility model has do with movement pattern of mobile station. 


\subsection{The Handover Channel Exchange Scheme:}

Consider the handover region between these two adjacent cells shown schematically in fig 1, below. Here the two cells are physically separated by the boundary line b12, the region between the two lines b1 and b2 represents the handover area. Beyond the right of line $\mathrm{b} 1$, the radio power received by a mobile from base station Bs is not sufficient to guarantee error- free communication.

The notation $\mathrm{M}_{\mathrm{i}}(\mathrm{j}, \mathrm{K})$ means the $\mathrm{i}$-th mobile holding a channel belonging to base station $\mathrm{Bs}_{\mathrm{j}}$ and moving towards the base station, $\mathrm{Bs}_{\mathrm{k}}$

Suppose at the time corresponding to figure $\mathrm{I}$ both $\mathrm{Bs}_{1}$, and $\mathrm{Bs}_{\mathrm{z}}$ have no free channel available, then, if the situation continues, after a certain period of time, the mobile $\mathrm{M}_{1}(2,1)$ will cross b2 and result in a handover failure, and its channel is released in cell 2. The $\mathrm{Bs}_{2}$ can then assign this channel to the mobile $\mathrm{M}_{2}(1,2)$ if the later is still in the handover area. The mobile are served in this manner in a conventional channel allocation scheme. In HCE scheme, however, the mobiles are allowed to exchange their channel if they are moving in opposite directions in the handover area. Thus, with the situation shown in figure 1 below, the channels held by the mobiles $\mathrm{m}_{2}(1,2)$ and $\mathrm{M}_{2}(2,1)$ are exchanged. This results in handover success for both mobiles instead of one as in the conventional scheme.

In HCE scheme, there is a close interaction between neighboring base stations. A base station $\mathrm{Bs}_{\mathrm{i}}$ queued a handover request from a mobile moving from cell $\mathrm{j}$ to cell $\mathrm{i}$ in a separate queue $\mathrm{Q}_{\mathrm{ij}}$. Similarly, the base station $\mathrm{Bs}_{\mathrm{j}}$ maintains a queue $\mathrm{Q}_{\mathrm{ji}}$ for handover request from mobiles moving from cell $\mathrm{i}$ to cell $\mathrm{j}$. The two base stations Bsi and Bsj together blocked if free channels are not available. However, a free channel is assigned new call request only if the handover request queue is empty. Thus, in the HCE scheme, as in other

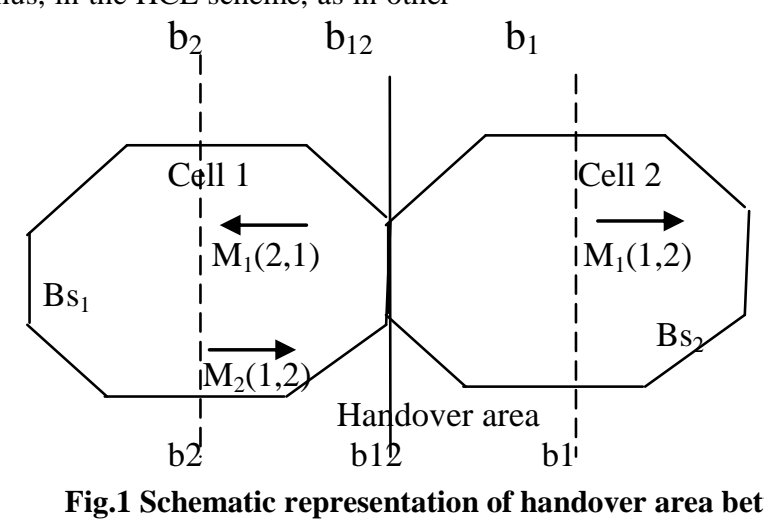

Fig.1 Schematic representation of handover area between two cells. process the queues $\mathrm{Q}_{\mathrm{ij}}$ so as to result in exchange of channels for suitably prioritized entries in the queues. It can be seen that because of the channel exchange scheme, at no time can both $\mathrm{Q}_{\mathrm{ij}}$ and $\mathrm{Q}_{\mathrm{ji}}$ be non-empty.

A handover request from a mobile in cell $i$ to the base station of cell $\mathrm{j}$ is processed in any of the following three ways;

i) If a free channel is available in cell $\mathrm{j}$, it is assigned to the mobile, this result in handover success.

ii) If a free channel is not available in cell $j$ and $Q_{i j}$ is empty, then the handover request is enqueued in $\mathrm{Q}_{\mathrm{ij}}$.

iii) If a free channel is not available in cell $j$ and $Q_{i j}$ is nonempty, then the mobile is made to exchange its channel with the channel held by the mobile whose handover request has the highest priority in $\mathrm{Q}_{\mathrm{ij}}$.

Once queued, a handover request can lead to handover success if a channel is released in the corresponding cell or if a channel is exchanged with a mobile of that cell. However, if a channel is not made available to a handover request within the internal during which it traverses the handover area, it results in a handover failure. The queued handover requests are periodically prioritized based on radio power measurements received from the mobiles. At any time if a free channel is available it is assigned to the highest priority mobile. Also if possible, a channel exchange is initiated for the highest priority in the queue. Timed-out handover requests, which correspond, to handover failures are periodically deleted from the queue. New call requests are just handover prioritization scheme a handover request has a higher priority than new call requests. 


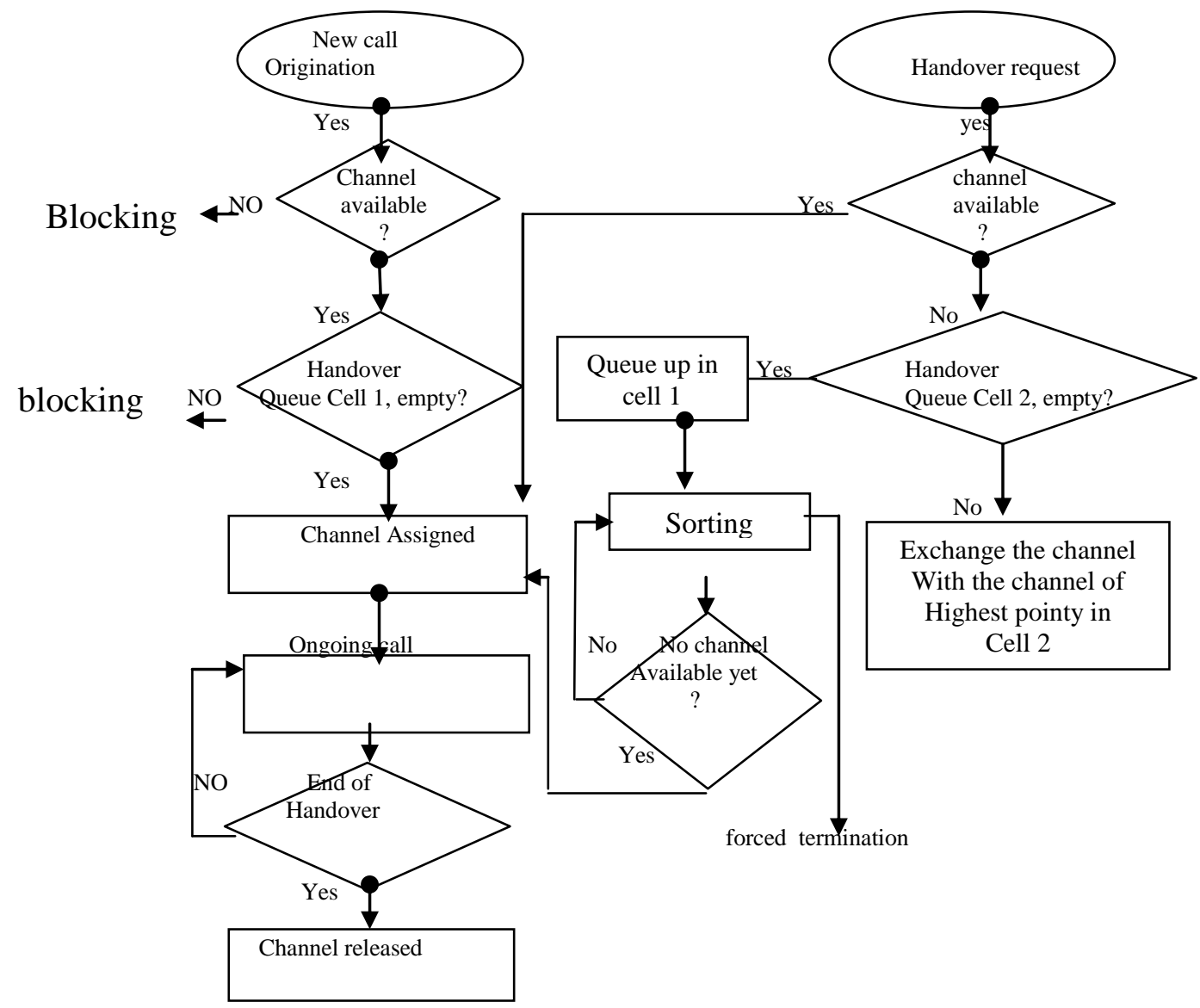

Fig 2: call flow chart for the HCE scheme

We begin by defining key terms for the model the dwell time (or the cell residence time) is the time over which a call may be maintained within a cell without handover. The holding time is the total duration of a call and is assumed to be exponentially distributed.

$\lambda_{n:}$ New call arrival rate

$\lambda_{h: \text { Handover call arrival rate }}$

$P_{n}$ : New call blocking probability

$P_{h}$ : Handover failure probability

$1 / \mu$ : mean cell residence time.

$1 / V$ : mean call holding time.

We define $p$ as the probability that the cell residence time of a call that has been assigned a channel expires before its call holding time.

$$
P=\frac{\mu}{\mu+V}
$$

A call is handed over if and only if its call holding time is greater than the cell residence time.

This event happens with probability $\mathrm{p}$. The probability that a new call is assigned one of the channels is $\left(1-p_{n}\right)$. Therefore $\lambda_{n}$ $\left(1-p_{n}\right)$ is the rate at which new calls are assigned one of the channels.

Suppose that a new call is generated in cell 1 , the probability that one of the channels is assigned to it is $\left(1-p_{n}\right)$ it is then handed over to cell 2 if its call holding time is greater than its cell residence time that is, with probability $\left(1-\mathrm{p}_{\mathrm{n}}\right) \mathrm{p}$.

The probability that call will not blocked during handover is $\left(1-p_{h}\right)$.
Therefore the probability that a new call (originating in cell 1) is successfully handed over from cell 1 to cell 2 is =

$\left(1-\mathrm{p}_{\mathrm{n}}\right) \quad\left(1-\mathrm{p}_{\mathrm{h}}\right) \mathrm{p}$

Based on the above argument and the fact that a call can be handed over several time before its natural termination, the following expression for $\lambda_{\mathrm{h}}$ can be obtained.

$$
\lambda_{h}=\frac{\lambda_{n}\left(1-P_{n}\right) P}{\left(1-P_{n}\right) P}
$$

The carried traffic here is $A_{c}$, and is a fraction of the offered traffic $\mathrm{A}_{\mathrm{t}}$ and is expressed as

$$
A_{c}=A_{t}\left(1-P_{n}\right)
$$

$P_{n}=\frac{\text { Number of blocked calls }}{\text { Number of new calls }}$

The offered traffic is expressed as

$$
\mathrm{A}_{\mathrm{t}}=\lambda_{\mathrm{n}} / \mu
$$

Where $\lambda_{n}$ is a new call arrival rate and $\mu$ is an average call duration

$$
P_{h}=\frac{\text { Number of calls being forced to terminate }}{\text { Number of successful new calls }}
$$

\section{SIMULATIONS AND RESULT}

Using the expressions derived above, the variation of $\lambda_{n}$ for channel exchange is obtained and compared with that of no channel exchange data obtained from STARCOMM 
NIGERIA LTD, and the simulation is done in MATLAB platform and the results presented below.

Table 1: Parameters for simulation

\begin{tabular}{|l|l|}
\hline Number of channels $(\mathrm{c})$ per Bs & 20 \\
\hline Handover queue $(\mathrm{k})$ & 3 \\
\hline New call arrival rate $\lambda_{\mathrm{n}}$ & $1 \mathrm{call} / \mathrm{sec}$ \\
\hline Mean cell residence time $1 / \mu$ & $60 \mathrm{sec}$ \\
\hline Mean call holding time $1 / \mathrm{v}$ & $120 \mathrm{sec}$ \\
\hline
\end{tabular}

Table 2: shows the new call arrival rate, new call blocking probability, handover failure probability the no channel exchange as gotten from STARCOMMS NIGERIA

\begin{tabular}{|l|l|l|l|l|}
\hline S/N & $\begin{array}{l}\text { New } \\
\text { Call } \\
\text { arrival } \\
\text { rate }\end{array}$ & $\begin{array}{l}\text { New call } \\
\text { blocking } \\
\text { probability }\end{array}$ & $\begin{array}{l}\text { Handover } \\
\text { failure } \\
\text { probability }\end{array}$ & $\begin{array}{l}\text { Handover } \\
\text { call arrival } \\
\text { rate(lambda) }\end{array}$ \\
\hline 1 & 0 & 0 & 0 & 0 \\
\hline 2 & 0.02 & 0 & 0 & 0.021 \\
\hline 3 & 0.04 & 0 & 0 & 0.04 \\
\hline 4 & 0.06 & 0.00625 & 0.000050 & 0.05 \\
\hline 5 & 0.08 & 0.01250 & 0.000159 & 0.07 \\
\hline 6 & 0.10 & 0.02500 & 0.000310 & 0.09 \\
\hline 7 & 0.12 & 0.05000 & 0.000385 & 0.123 \\
\hline 8 & 0.14 & 0.08750 & 0.000598 & 0.128 \\
\hline 9 & 0.16 & 0.12975 & 0.001186 & 0.14 \\
\hline 10 & 0.18 & 0.21900 & 0.001537 & 0.172 \\
\hline 11 & 0.20 & 0.25000 & 0.003000 & 0.19 \\
\hline
\end{tabular}

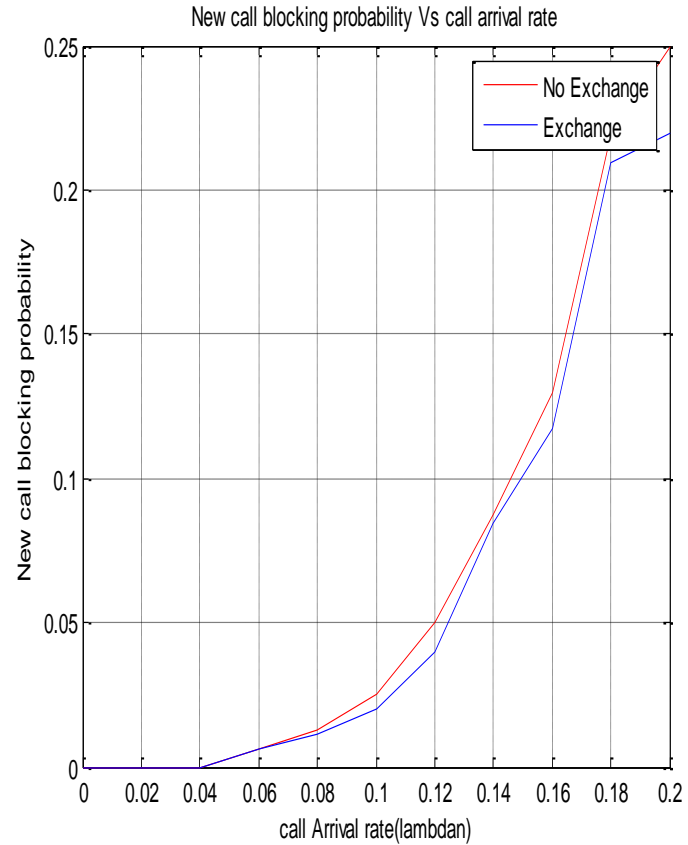

Figure 3 New call blocking probability Vs call arrival rate

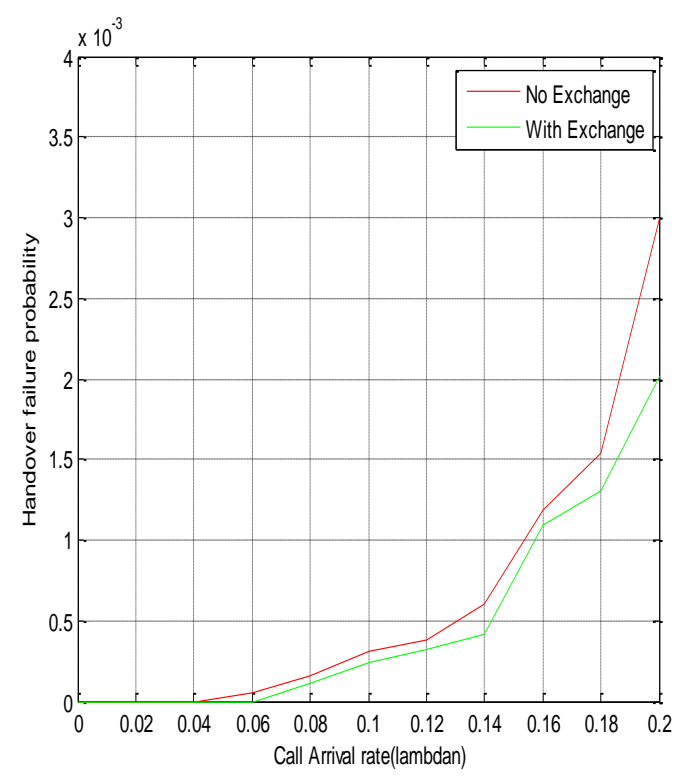

Figure 4 Handover failure probability $\left(P_{h}\right)$ versus arrival rate $\left(\lambda_{n}\right)$ of new calls in a cell. 


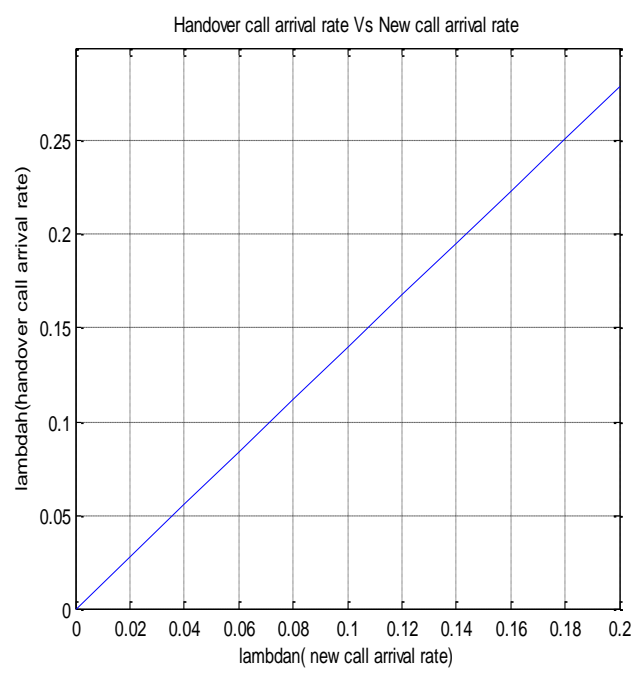

Figure 5 handover call arrival rate Vs new call arrival rate

Fig. 3 shows a plot of the new call Blocking probability $\left(\mathrm{p}_{\mathrm{n}}\right)$ versus the arrival rate. The plots are shown for both channel exchange and no channel exchange scheme. It is clear from the plot that $p_{n}$ increases with $\lambda_{n}$. But the channel exchange scheme produces a lower Blocking probability for each value of $\lambda_{n}$ showing that with this scheme improvement in new call blocking probability is achieved.

Fig. 4 Also show the plot of Handover failure probability $\left(\mathrm{P}_{\mathrm{h}}\right)$ versus arrival rate $\left(\lambda_{\mathrm{n}}\right)$ of new calls in a cell. For both channel exchange and No channel exchange with an increase in $\lambda_{\mathrm{n}}$ (the system load), the handover failure probability increases as expected but the channel exchange scheme performs better than the handover scheme with no exchange of channel.

Fig 5 shows accordingly the improvement of the call arrival rate by the channel exchange scheme.

\section{CONCLUSION}

The handover channel exchange scheme presented in this work improves handover success rate by exchanging channels between two mobiles moving in opposite directions across the handover area of adjacent cells. The improvement in cellular system performance by the use of the new scheme has been demonstrated by simulation of call traffic in two adjacent cells of a mobile cellular system.

The simulation results show that the new scheme greatly minimizes handover failure probability with no detrimental effect on call blocking probability.

\section{REFERENCES}

[1] T. Salih and K. fidanboylu", performance Analysis and, modeling of two- tier cellular Networks with queuing Handoff calls," proc of the $8^{\text {th }}$ IEEE symposium on computers and communication (15cc103), vol, pp217224.

[2] T. Salih and Fidanboylu, " A comparison of the performance of two-tier cellular networks based on queuing handoff calls", international journal of signal processing vo.1, 2004, No.1-4, pp,343-342.

[3] Y. Zhang and D.liu, "Adaptive algorithm for call Admission control in wireless Networks," IEEE Global Telecom conference (GLOBECOM' 01), Vol 6,2001, pp.3628-3632.

[4] S. Choi and K. Sohraby, "Analysis of a mobile cellular systems with Hand-off priority and Hysteresis control" IEEE INFOCOM 2000, Vol.1, March 2000 .pp.217-224

[5] Alexe E. Leu and Brian L. Mark, “ Modeling and Analysis of fast Handoff Algorithms for Microcelliuar Networks," proceedings of the $10^{\text {th }}$ IEE MASOTS 2002 Oct 2002, pp.321-328.

[6] A Jera, A. Molinaro and S. Marano," Handoff management with mobility Estimation in Hierarchical systems," IEEE Transaction on vehicular Technology vol.51, Sept 2002, pp.915-934.

[7] S. Tekinay and B. Jabbari, " a measurement based prioritization scheme for handovers in mobile cellular network “ IEEE journal on selected areas in communications, 1101 10,N08,oct 1992 pp,1343-1350.

[8] P. Agrawal, Dinesh K. Anvekar and B. Narendra, “ channel management policies for Handovers in cellular Networks," Bell labs Technical Journal, No1.1, Autumn, 1996,pp96-109.

[9] Salsano, S., Polidoro, A., Mingardi, C., Niccolini, S., and Veltri, L. (2008). SIP-basedmobility management in next-generation networks. IEEE Wireless Communications, Vol 15, No 2, April 2008, pp. 92-99.

[10] Wei Kuang Lai, and Jung Chia Chiu "Improving Handoff Performance in Wireless OverlayNetworks by Switching Between Two-Layer IPv6 and One-Layer IPv6 Addressing,'IEEE Journal on selected areas in communications, Vol. 23, No. 11, November 20052129

[11] A. Mishra, M. Shin, and W. Arbaugh, "An empirical analysis of the IEEE 802.11 MAC layer handoff process," SIGCOMM Comput.Commun. Rev., vol. 33, no. 2, pp. 93-102, 2003. 